\title{
Ralinepag Reduces Pulmonary Vascular Resistance (PVR) in a Phase 2 Study Confirming Preclinical Findings on Prostacyclin (IP) Receptors in Human Tissues
}

Authors: loana R. Preston ${ }^{1}$, Lei Shen², Jigisha Patel ${ }^{2}$, Namita Sood ${ }^{3}$, Fernando Torres ${ }^{4}$, Linda Cadaret ${ }^{5}$, John Adams ${ }^{6}$, Lucie H. Clapp ${ }^{2}$

Affiliations: ${ }^{1}$ Tufts Medical Center, Pulmonary and Critical Care, Boston, Massachusetts, USA; ${ }^{2}$ Institute of Cardiovascular Science, University College London, London, UK; ${ }^{3}$ University of Texas Health Science Center of Houston, Houston, TX, USA; ${ }^{4}$ Medical Director of Lung Transplantation, Head of Pulmonary Hypertension Program, UT Southwestern Medical Center, Dallas, TX, USA; ${ }^{5}$ Division of Cardiology, University of lowa, lowa City, lowa; ${ }^{6}$ Arena Pharmaceuticals, Inc., San Diego, CA, USA

\section{Purpose}

Ralinepag is a next-generation, selective and potent IP receptor agonist in development for pulmonary arterial hypertension (PAH). Human IP receptor binding affinity and selectivity of ralinepag were examined, and its functional receptor activation was compared to that of selexipag and iloprost on human platelets, pulmonary artery smooth muscle cells (PASMCs), and pulmonary arteries (PAs). To confirm preclinical efficacy, change in PVR was evaluated in patients with functional class (FC) II-IV group 1 PAH on disease-specific background therapy in a Phase 2 study.

\section{Methods}

Potency, selectivity, and functional assays were conducted, including cAMP accumulation as well as cell proliferation in human IP receptor-expressing Chinese hamster ovary $(\mathrm{CHO})$ cells and human PASMCs from $\mathrm{PAH}$ patients. Human platelet responses were measured by light transmittance aggregometry. Distal PAs were mounted in a myograph and preconstricted with U46619. In the Phase 2 study, adults with stable FC II-IV PAH were randomized 2:1 to receive ralinepag $(n=40)$ or placebo $(n=21)$ for 9 weeks of titration (up to $300 \mathrm{mg}$ twice daily), then 13-week maintenance of maximum tolerated dose. Right heart catheterization was performed at baseline and at 22 weeks. The primary efficacy endpoint was change in PVR from baseline to week 22.

\section{Results}

Ralinepag has high binding affinity $\left(K_{i}=0.003 \mu \mathrm{M}\right)$ and selectivity at the human IP receptor. Ralinepag, iloprost, and MRE-269 (selexipag metabolite) increased cAMP in IP receptor-expressing CHO cells; ralinepag and MRE-269 had an $\mathrm{E}_{\mathrm{Max}}$ of $67 \%$ and $48 \%$ relative to iloprost. Ralinepag inhibited PASMC proliferation and human ADP-stimulated platelet aggregation more potently than MRE-269. In PAs, ralinepag caused greater relaxation than iloprost and MRE-269. The Phase 2 clinical study enrolled patients with $\mathrm{FC} \mathrm{II/III/IV} \mathrm{(56 \% /43 \% /2 \% )} \mathrm{PAH} \mathrm{and} \mathrm{mean} \mathrm{6-minute} \mathrm{walk} \mathrm{distance} \mathrm{of} 378 \mathrm{~m}$. Baseline median PVR was 705 (ralinepag) and 480 (placebo) dyn.s. $\mathrm{cm}^{-5}$; all patients were on background PAH treatment, with $65 \%$ (ralinepag-treated) and $48 \%$ (placebo) of patients receiving dual therapy. Ralinepag reduced median PVR by 163.9 dyn $\cdot \mathrm{s} \cdot \mathrm{cm}^{-5}$ from baseline, versus a 0.7 dyn $\cdot \mathrm{s} \cdot \mathrm{cm}^{-5}$ increase with placebo $(P=0.02)$.

\section{Conclusions}

Ralinepag is a next-generation, selective and potent IP receptor agonist that elicited greater pharmacological responses in human platelets, PAs, and PASMCs than the active selexipag metabolite, MRE-269, and had a favorable vasorelaxant profile compared with iloprost. In a Phase 2 clinical study, ralinepag significantly reduced PVR vs placebo in PAH patients on background therapy, confirming preclinical findings and providing rationale for further investigation.

\section{Clinical Implications}

The Phase 2 study of ralinepag in patients on background therapy showed significant improvement in PVR, a well-established indicator of treatment benefit that correlates with long-term clinical outcomes in patients with PAH.

Character count: (section labels not included) $=\mathbf{2 8 9 1}$ of 2900 allowed 